\title{
Information Flow in Color Appearance Neural Networks ${ }^{\dagger}$
}

\author{
Jesús Malo \\ Image Processing Lab, Universitat de València, Valencia, Spain \\ + Presented at the Entropy 2021: The Scientific Tool of the 21st Century, 5-7 May 2021; Available online: \\ https://sciforum.net/conference/Entropy2021/.
}

Published: 5 May 2021

Color Appearance Models are biological neural networks that consist of a cascade of linear + nonlinear layers that transform the measurements at the retina into an internal representation of color that correlates with psychophysical experience. The basic layers of these networks include: (1) chromatic adaptation (normalization of the mean and covariance of the color manifold), (2) change to opponent color channels (PCA-like rotation), and (3) saturating nonlinearities to get perceptually Euclidean color representations (dimension-wise equalization). The Efficient Coding Hypothesis in neuroscience argues that these transforms should emerge from information theory [Barlow Proc.Nat.Phys.Lab.59, Barlow Network 01]. In the specific case of color vision there are a number of evidences of this [Buschbaum Proc.Roy.Soc.83, Twer Network 01, Laparra\&Malo Neural Comp. 12, Laparra\&Malo Front.Human.Neurosci.15]. The question for these color networks is, what is the coding gain due to the different mechanisms in the networks?

In this work, representative Color Appearance Models are analyzed in terms of how they modify the statistical redundancy along the network and how much information is transferred from the input to the noisy response. The proposed information-theoretic analysis is done using methods and data that were not available before: (1) new statistical tools to estimate (multivariate) information-theoretic quantities between multidimensional sets based on Gaussianization [Laparra\&Malo IEEE Trans.Neur.Nets.11, Johnson, Laparra \& Malo ICML 19], and (2) new colorimetrically calibrated scenes in different CIE illuminations for proper evaluation of chromatic adaptation [Gutmann,Laparra, Hyvarinen \& Malo PLOS 14].

Results identify the psychophysical mechanisms critically responsible for gains in chromatic information transference: opponent channels and their nonlinear nature are more important than chromatic adaptation at the retina. Moreover, our visual neural pathway allocates at least $70 \%$ of the information capacity for spatial information as opposed to only $30 \%$ devoted to color.

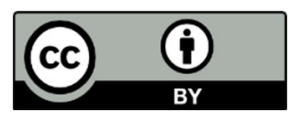

(C) 2021 by the author. Licensee MDPI, Basel, Switzerland. This article is an open access article distributed under the terms and conditions of the Creative Commons Attribution (CC BY) license (http://creativecommons.org/licenses/by/4.0/). 